\title{
Open access community support groups for people with personality disorder: attendance and impact on use of other services
}

\author{
Steve Miller, ${ }^{1}$ Mike J. Crawford ${ }^{2}$
}

The Psychiatrist (2010), 34, 177-181, doi: 10.1192/pb.bp.109.026575

${ }^{1}$ Department of Psychotherapy, Springfield University Hospital, London; ${ }^{2}$ Department of Psychological Medicine, Imperial College London

Correspondence to Steve Miller (Stephen.miller@swlstg-tr.nhs.uk)

\begin{abstract}
Aims and method To describe a new open access community service for people with personality disorder and to explore interim service utilisation and outcomes. Routine data were analysed together with those from a cross-sectional survey.

Results During the first 16 months of the service, 171 people attended, of whom 142 $(83.0 \%)$ returned on at least one other occasion. The median number of attendances was seven (IQR $=3.0-22.0)$. Over $90 \%$ of responders to the survey met criteria for 'probable personality disorder' and levels of social dysfunction were high. Presentations to emergency services, contacts with other services and in-patient admissions were reduced. Social functioning improved.
\end{abstract}

Clinical implications This service attracted a large number of people with significant health and social problems. Use of the service was associated with improved social functioning and reduced use of other services.

Declaration of interest None.
As part of a series of initiatives aimed at improving services for people with personality disorder the Department of Health funded a series of community-based pilot services that were set up in 2004/2005. ${ }^{1}$ In south-west London, one of these services, the Service User Network (SUN) project, was designed to offer community-based open access support groups for people with personality disorder. It aimed to help people develop effective ways of coping, reduce emergencies and improve access to appropriate services. A fundamental principle of the project was the utilisation of service users' own resources for support and a commitment to formally include them as an integral part of service delivery. Service users were employed in the design and early management of the project, and service users work within the team as both volunteers and as paid full team members.

We set out to record levels of attendance, examine characteristics of people utilising the service and explore the impact of the service on use of other medical and Social Services, social functioning and satisfaction with care. We hypothesised that contact with the SUN project would reduce use of in-patient mental health and emergency medical services and increase appropriate contacts with other services.

\section{Method}

\section{Study design}

Routine records kept demographic details of all those who attended a group. These, together with subsequent levels of group attendance, were combined with a cross-sectional survey of a subsample of service users who had been with the service for at least 6 months.

\section{The intervention}

Information about the project was publicised via local health, social care and voluntary services. Entry to the SUN project were solely by self-referral. All clinical work in the SUN project takes place in groups. At their first attendance new potential members are asked to complete a crisis and support plan in a group with existing SUN project members. This document lists, from the service users perspective, all of the crises that they encounter in their life together with any steps, identified within the group as a whole, which might be taken to support the individual and prevent these crises from becoming emergencies. The document performs a dual function of assessment and engagement with the process of improving coping effectiveness. It provides information concerning risky behaviours including selfdamaging and behaviours that might damage others together with the precipitants of such behaviours. Once the crisis and support plan is completed the individual becomes a member of the SUN project and is free to attend all groups.

The SUN groups meet either three or four times per week for $2.5 \mathrm{~h}$ and although always concerned with support, these groups have a variety of other named functions such as: social groups, business meetings, crisis and support planning groups, and support groups. Groups focus on crises and support and employ a structured cognitive approach focusing upon the modification of appraisal to improve 
coping. Careful attention is given to the systematic incorporation of material from outside group time. Within this framework, practical supportive activities can either be planned for outside the group or where possible take place during the group. Whenever an individual attends a group in a crisis practical action is taken within the group to resolve it. Members of the SUN project may call upon each other for out-of-hours support, and have arranged some groups independent of healthcare staff. Previous reports have highlighted the importance that many people with personality disorder attach to support from peers. ${ }^{2}$ The SUN project seeks to improve safety and the effectiveness of this process by formalising it. A similar method of support has been described in detail by Higgins. ${ }^{3}$

In order to provide a service for a large geographical area, four separate local groups were set up over an 8-month period. At the time of the survey the first group to be set up had been in operation for 18 months and the other three groups had been in operation for 15, 12 and 9 months respectively.

\section{Data collection}

Data on age, gender and ethnicity were collected as part of the registration process. Remaining data were collected as part of a survey of SUN members conducted in October 2006. Questionnaires were sent out together with a stamped addressed envelope. All those who did not return the survey within 2 weeks were written to again. All those who did not return the survey after the second letter were telephoned. The survey comprised an assessment of the following.

1 Personality disorder: using the Standardised Assessment of Personality - Abbreviated Scale. ${ }^{4}$ This is an eight-item screening questionnaire for which a score of three or more provides a valid indicator of the likely presence of personality disorder.

2 Social functioning using the eight-item Social Function Questionnaire (SFQ). ${ }^{5}$

3 Service utilisation: numbers of contacts with primary and secondary healthcare services, social care and housing services, probation services, emergency medical services, in-patient mental health services and telephone helplines. As a proxy for appropriate and emergency care respectively, respondents were asked to state whether the contact had been planned or unplanned.

4 Subjective experience of satisfaction with the project and impact of the service using a ten-item questionnaire specifically designed for the study (details available from the author).

5 Finally, respondents were asked if they were still using the service. Those that told us they were no longer using it were asking to state their reasons for leaving.

Items 1-4 were selected in order to facilitate comparison with data collected by other community-based services for people with personality disorder. ${ }^{6}$ Items on the satisfaction scale were suggested for inclusion by members of the SUN project. For items 2 and 3, respondents were asked to provide information on the 6 months before joining SUN and 6 months after their first contact with the service. Ethics committee approval for the study was obtained prior to the start of data collection.

\section{Data analysis}

All data were entered onto SPSS (Version 15.0) for Windows for data analysis. The distribution and central tendency of variables were examined using standard deviations for normally distributed continuous data, and interquartile range (IQR) for non-parametric data. Appropriate univariate statistical tests (paired $t$-tests and Wilcoxen matched pairs tests) were used to examine changes in service utilisation and other outcomes.

\section{Results}

During the first 16 months of the service, 765 groups were held. A total of 171 people attended at least one group, completed a crisis and support plan, and became a member of the SUN project. Of these, $116(67.8 \%)$ were women and $55(32.2 \%)$ were men. Age was stated by 116 members and this ranged from 22 to 62 (mean 39.7, s.d.= 9.6). Information on ethnicity was provided by 132 people $(77.2 \%)$ and $109(63.7 \%)$ were White British, 13 (7.6\%) were White 'other' and $10(5.8 \%)$ were from Black and minority ethnic communities.

Of the 171 members, $142(83.0 \%)$ returned to the group on at least one other occasion. The median number of groups attended was seven $(\mathrm{IQR}=3.0-22.0)$. Attendance patterns were irregular, 51 people did not attend for a period of 1 month or more then returned to the project, and during periods of attendance the ratio of groups attended to groups not attended was 4 to 1 . Men were as likely as women to attend on more than one occasion (32.4\% compared with $\left.31.0 \%, \chi^{2}=0.20, P=0.89\right)$ and members from Black and minority ethnic communities were as likely to return as those from British White backgrounds (8.0\% of those who returned were from Black and minority ethnic communities as were $5.0 \%$ of those who did not). Mean age of the two groups was also similar (39.6 among those attending more than once and 41.1 among the remainder). At the time of the survey, $141(82.5 \%)$ of the 171 had been in contact with the service for at least 6 months and were therefore sent a copy of the survey questionnaire. Of these, 61 (43.3\%) completed the survey. Those who completed the survey were similar in terms of gender and ethnicity but were younger than those who did not complete it (mean age 37.0 years compared with 41.2, $t=5.31, P=0.02$ ) and had attended a greater number of groups (15 compared with $6, Z=-4.25, P<0.001$ ).

Forty-eight (78.8\%) of those who completed the survey filled out the abbreviated version of the Standardised Assessment of Personality. Their mean score was 5.3 (s.d.=1.8), with $44(91.7 \%)$ having a score of 3 or more, suggesting 'probable personality disorder'.

\section{Service utilisation}

Levels of self-reported service utilisation were obtained from all 61 people who completed the survey. Changes in service utilisation in the 6 months prior to and following SUN membership are presented in Table 1 . None of the 61 service users reported contacts with probation services during this period. Reductions in use of all services were seen following contact with the SUN project with the exception of use of telephone helplines, for which there was a slight increase. Reductions in contact with primary care, mental 


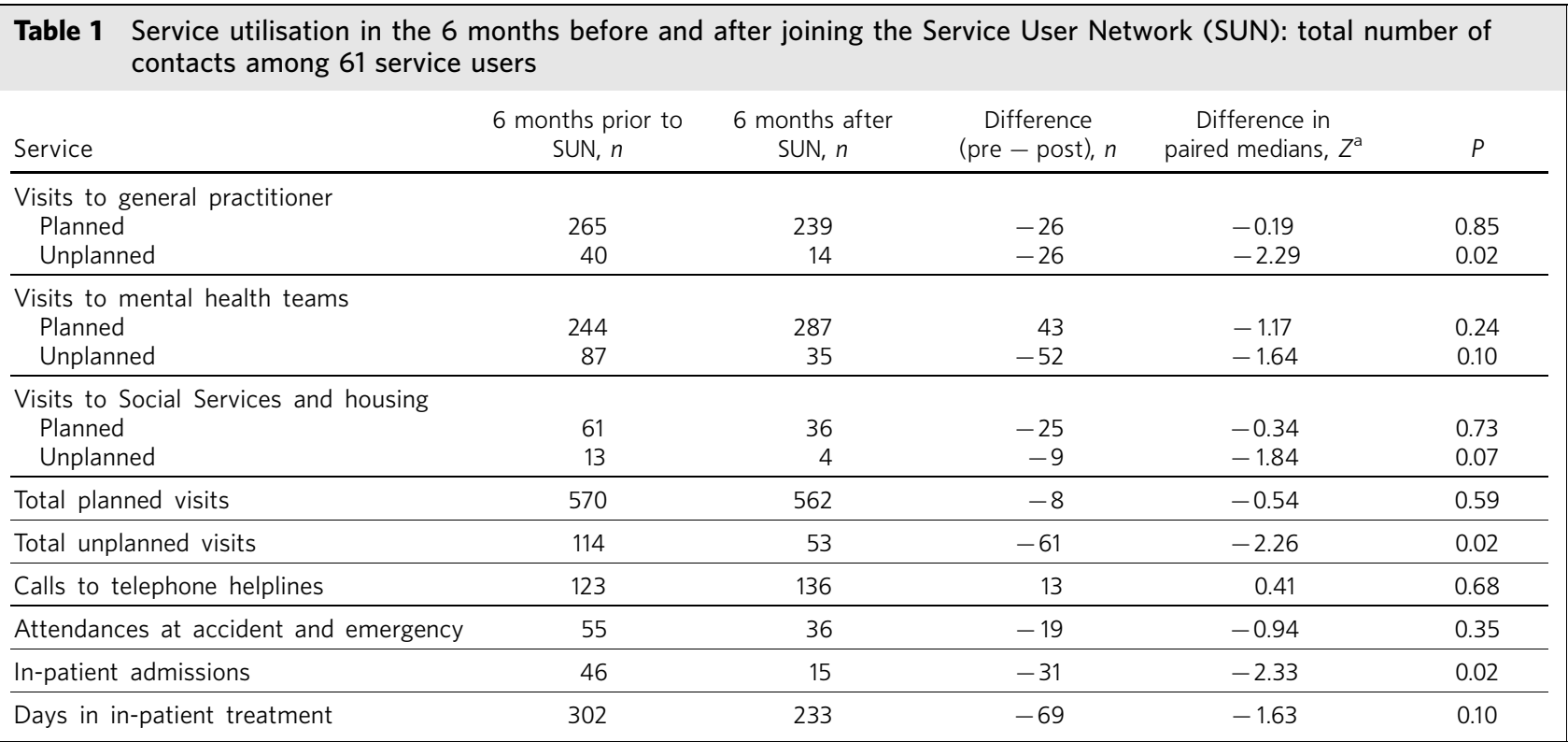

a. Wilcoxon paired-rank test.

health and Social Services were greater for unplanned contacts than for planned ones. The ratio of planned to unplanned contacts increased from 5 to 1 in the 6 months prior to joining SUN to 10.6 to 1 in the 6 months after joining.

\section{Social function and satisfaction with services}

Although $48(78.7 \%)$ respondents filled out part of the SFQ, only $42(68.9 \%)$ fully completed it for both the pre- and post-treatment period. The mean SFQ score was 14.0 (s.d.=4.4) in the period prior to joining SUN and 12.1 (s.d. =6.0) in the period after. The difference in mean SFQ scores before and after having contact with SUN among these 42 people was 2.0 (95\% CI $0.47-3.53, P=0.01)$. Fifty-three (86.9\%) of those who completed the survey responded to questions on satisfaction with the SUN project and the impact of the service. Their responses are presented in Table 2.

\section{Leaving the service}

Twenty-seven (44.3\%) people who responded to the survey stated that they had left the service. The most frequently stated reason for leaving the service was that other commitments made it difficult to attend $(n=8,29.6 \%)$. Four leavers $(14.8 \%)$ stated that they no longer attended because of 'difficulties with other group members', and three $(11.1 \%)$ that they had found the service unhelpful. Other reasons for leaving included moving away from the area and being admitted to hospital. Those who had left were similar to those who remained with the service expect that the former tended to report better social functioning (SFQ mean score 10.4 compared with 13.6, $t=3.09, P=0.08$ ).

\section{Discussion}

The SUN project was set up to deliver an innovative, deinstitutionalised approach to helping people with personality disorder develop better ways of coping with
Table 2 Satisfaction with the Service User Network (SUN): responses of 53 people using the service

\begin{tabular}{lcc} 
Statement & $\begin{array}{c}\text { Mean } \\
\text { score }^{\mathrm{a}}\end{array}$ & s.d. \\
\hline I feel more supported & 3.4 & 1.1 \\
\hline I feel more included & 3.4 & 1.2 \\
\hline I am learning more coping strategies & 3.4 & 1.2 \\
\hline I feel more empowered & 3.2 & 1.1 \\
\hline $\begin{array}{l}\text { I am having fewer crises that become } \\
\text { emergencies }\end{array}$ & 3.3 & 1.2 \\
\hline $\begin{array}{l}\text { My difficulties are becoming easier to } \\
\text { manage }\end{array}$ & 3.1 & 1.1 \\
\hline $\begin{array}{l}\text { I am now able to access other services } \\
\text { more simply }\end{array}$ & 3.0 & 1.1 \\
\hline $\begin{array}{l}\text { The description of each group is easy to } \\
\text { understand }\end{array}$ & 3.8 & 0.9 \\
\hline I feel more able to talk/express myself & 3.4 & 1.2 \\
\hline I am satisfied with the SUN project & 3.2 & 1.1 \\
\hline
\end{tabular}

a. 1, strongly disagree; 5 , strongly agree.

b. 51 people responded to this question.

crises. These data show that nearly all SUN members who returned questionnaires had probable personality disorder and had high levels of social dysfunction equivalent to those presenting to emergency services for treatment of mental disorder. ${ }^{5}$ Decreased levels of contact with health and social care services in the period after joining the SUN project were reported and this was particularly marked for 'unplanned' contacts with services and use of in-patient treatment. Higher levels of social functioning in the 6 months following contact with the service than in the prior 6 months were reported, the reduction in SFQ score being both clinically significant and larger than that reported in previous studies of out-patient psychological treatment for people with personality disorder. ${ }^{78}$ 
Care needs to be taken interpreting these findings, however, because of a number of important methodological limitations. First, all the data we collected were self-reported and it is possible that response bias resulted in some or all of the differences we found. Self-report data may lead to an overdiagnosis of personality disorder when compared with structured interviewing. ${ }^{9}$ Although data on service utilisation could have been cross-checked against routine records we did not have ethical committee approval to obtain these data and had to rely on self-report. The other main limitation is the response rate. Nevertheless, $43 \%$ is better than response rates for postal surveys among people with both personality disorder $^{10}$ and depression. ${ }^{11}$ It may have been possible to reduce the amount of missing data by integrating the completion of questionnaires into group sessions. However, we took the view that such an approach may have undermined the group aims of trying to strengthen peer support and placing less emphasis on the role of service providers. The net result is that our data cannot be extrapolated with certainty to those not returning questionnaires.

One of the methodological strengths of the project was that we used measures that have been widely used in other studies of people with personality disorder including a national evaluation of ten other community-based services in England. Thus these findings can be compared with those from other services that delivered more traditional approaches to helping people with personality disorder. ${ }^{12}$ Age and gender of SUN members is similar to that of other people using community-based services for people with personality disorder. For instance, women made up twothirds of all SUN members, and although men are as likely to have personality disorder as women ${ }^{13}$ the preponderance of women among SUN members is in keeping with those of other community-based services for people with personality disorder. $^{12}$ The higher proportion of people from Black and minority ethnic communities using the SUN project reflects the multi-ethic population in the local area. Over $90 \%$ of SUN members who returned questionnaires had probable personality disorder. Although this is also true of people with personality disorder accessing other new personality disorder services, the high levels of personality disturbance and social dysfunction we found are interesting given that all those who accessed the service were self-referrals. Providers of other personality disorder services have expressed concerns that opening them up to self-referral may mean that people with low levels of need access the service, ${ }^{6}$ but we found no evidence to support this notion in this survey.

Providers of mental health services have been told that it is unacceptable to withhold services from people on the basis of their having a personality disorder. Given that many service providers do not currently offer specialist personality disorder services, and may not have access to new funding, the development of specialist personality disorder services is a considerable challenge. General population surveys suggest that approximately $5 \%$ of people may have a personality disorder, ${ }^{13}$ but most interventions for people with personality disorder developed to date offer intensive services over relatively long periods of time and therefore tend to be offered to only a small minority of those that may potentially benefit from them. ${ }^{14}$ The SUN project delivers a different kind of service aimed at enhancing opportunities for peer support and helping people with personality disorder make better use of existing services. Providing open access allows people to utilise the service at a time of their own choice and broadens the scope for support to those who are only able to attend in an irregular manner. Such a service has the potential to be offered to many more people than traditional psychotherapy services.

We believe that one of the strengths of the service was that service users were involved during the development phase of the project and also contributed to the design of the patient survey. Service users were instrumental in essential aspects of the project such as publicity design, setting group rules for acceptable behaviour and liaising with other services. This generated a respectful collaboration that fostered a sense of inclusion and empowerment leading to a positive reappraisal of the resources for support that service users themselves possessed.

The exploratory nature of this study, together with the limitations in the study design mean that we cannot be certain what the true costs and benefits of this service are. However, at a cost of only $£ 280000$ per year during the early years of its development the service is relatively inexpensive and the reductions in the use of other services by people using the SUN project during this period suggest it may be a cost-effective means of helping people with personality disorder. Future research should aim to collect longitudinal data from service users and to combine these with data from other health and social care services on actual levels of service contact. A randomised trial of a new service run elsewhere, along similar lines to the SUN project, could generate high-quality data on the effects and cost-effectiveness of support groups for people with personality disorder.

\section{About the authors}

Steve Miller is a Consultant Psychiatrist in Psychotherapy and the Clinical Lead for Personality Disorder at the South West London \& St George's Mental Health NHS Trust, Springfield University Hospital, London. Mike J. Crawford is a Reader in Mental Health Services Research at the Department of Psychological Medicine, Imperial College London.

\section{References}

1 Haigh R. The 16 personality disorder pilot projects. Ment Health Rev J 2007; 12: 29-39.

2 Faulkner A, Gillespie S, Imlack S, Dhillon K, Crawford M. An inclusive project: user-led research into services for people with a diagnosis of personality disorder. Ment Health Today 2008; February: 24-7.

3 Higgins B. Does anyone feel they need support tonight? 24 hour care on a day unit. Ther Communities 1997; 18: 55-61.

4 Moran P, Leese M, Lee T, Walters P, Thornicroft G, Mann A Standardised Assessment of Personality - Abbreviated Scale (SAPAS): preliminary validation of a brief screen for personality disorder. Br J Psychiatry 2003; 183: 228-32.

5 Tyrer P, Nur U, Crawford M, Karlsen S, McLean C, Rao B, et al. The Social Functioning Questionnaire: a rapid and robust measure of perceived functioning. Int J Soc Psychiatry 2005; 51: 265-75.

6 Crawford MJ, Rutter D, Price K, Weaver T, Josson M, Tyrer $P$, et al. Learning the Lessons: A Multi-Method Evaluation of Dedicated CommunityBased Services for People with Personality Disorder. National Coordinating Centre for Service Delivery and Organisation Research Programme, 2007. 
7 Davidson K, Norrie J, Tyrer P, Gumley A, Tata P, Murray H, et al. The effectiveness of cognitive behavior therapy for borderline personality disorder: results from the borderline personality disorder study of cognitive therapy (BOSCOT) trial. J Personal Disord 2006; 20: 450-65.

8 Huband N, McMurran M, Evans C, Dugan C. Social problem-solving plus psychoeducation for adults with personality disorder. Pragmatic randomised controlled trial. Br J Psychiatry 2007; 190: 307-13.

9 Verheul R, Hartgers C, Van Den Brink W, Koeter MWJ. The effect of sampling, diagnostic criteria and Aassessment procedures on the observed prevalence of DSM-III-R personality disorders among treated alcoholics. J Stud Alcohol, 1998; 59: 227-36.

10 Blum N, Pfohl B, John DS, Monahan P, Black DW. STEPPS: a cognitivebehavioral systems-based group treatment for outpatients with borderline personality disorder-a preliminary report. Compr Psychiatry 2002; 43: 301-10.

11 Graham CR, Banrjee S, Gill RS. Using postal questionnaires to identify carer depression prior to initial patient contact. Psychiatr Bull 2009; 33: 169-79.

12 Crawford MJ, Price K, Gordon F, Josson M, Taylor B, Bateman A, et al. Engagement and retention in specialist services for people with personality disorder. Acta Psychiatr Scand 2009; 119: 304-11.

13 Coid J, Yang M, Tyrer P, Roberts A, Ullrich S. Prevalence and correlates of personality disorder in Great Britain. Br J Psychiatry 2006; 188: 423-31.

14 Rutter $D$, Tyrer $P$. The value of therapeutic communities in the treatment of personality disorder: a suitable place for treatment? J Psychiat Pract 2003; 9: 291-302.

\title{
Two consultants for one patient: service users' and service providers' views on 'New Ways'
}

\author{
Ankush Singhal, ${ }^{1}$ Deepak Garg, ${ }^{2}$ Alok Kumar Rana, ${ }^{3}$ Miriam Naheed ${ }^{2}$
}

The Psychiatrist (2010), 34, 181-186, doi: 10.1192/pb.bp.108.024208

${ }^{1}$ Lister Hospital, Stevenage; ${ }^{2}$ Furness General Hospital, Barrow-in-Furness;

${ }^{3}$ Royal Hampshire County Hospital, Winchester

Correspondence to Ankush Singhal (imdrankushsinghal@yahoo.com)
Aims and method To investigate, through a semi-qualitative survey at three geographical sites, health professionals' and service users' opinion about the impact of providing separate consultants for in-patient and community settings. It looked at the perceived affect on various issues such as the course of the illness, service delivery, patients' satisfaction as well as the skills and training of psychiatrists.

Results Opinion was divided about the level of satisfaction, advantages, consultants' skills and success of this model. The most consistent theme related to the problems with the continuation of care and therapeutic relationship. Most of the respondents were not fully informed about this change. An overwhelming majority believed that in-patient psychiatry is not a separate specialty.

Clinical implications Communication and the sharing of information between the two consultants is the key to success in this model.

Declaration of interest None.
New Ways of Working is about supporting and enabling consultant psychiatrists, among others, to deliver effective and person-centred care. Work on New Ways of Working started in 2003 when two national conferences for consultant psychiatrists (in March and April 2003) summarised the problems in the profession - significant difficulties in recruiting and retaining consultant psychiatrists because of the increasing demands of the role, increasing degrees of burnout among consultants, unsustainably high case-loads and crippling expenditure on agency locum doctors to try and plug the gaps. The National Steering Group, jointly chaired by the Royal College of Psychiatrists and the National Institute for Mental Health in England (NIMHE) explored and reviewed the role of psychiatrists and their interface with other mental health professionals. ${ }^{1}$ The NIMHE, the Department of Health and the Royal College of Psychiatrists jointly set up the New Ways of Working initiative to address these concerns.

One key aspect of New Ways of Working was the modification of the consultant psychiatrist's job as an in-patient specialist or 'working across the acute care pathway in crisis and in-patient work'. ${ }^{2}$ Many National Health Service (NHS) trusts have developed these roles as a part of the so-called 'functional model' in at least part of their area. $^{2}$ The functional model includes many changes, but its core feature is that consultants work either on the in-patient or the community side, with one specialist team rather than in the old-styled geographical sectors. Closely related to this functional division is the status of in-patient psychiatry. Currently, acute psychiatric in-patient care is one of the top priorities. ${ }^{3}$ Recently there has been a lot of 